\title{
British Society of Breast Radiology Annual Scientific Meeting 2012
}

\author{
Leeds, UK. 12-13 November 2012 \\ Published: 9 November 2012 \\ These abstracts are available online at http://breast-cancer-research.com/supplements/14/S1
}

\section{ORAL PRESENTATIONS}

\section{1}

Utility of MRI in low and low to moderate density breasts with

invasive lobular carcinoma

B Rengabashyam*, N Sharma, B Dall

The Leeds Teaching Hospitals NHS Trust, Leeds, UK

Breast Cancer Research 2012, 14(Suppl 1):O1

Objective: To determine the feasibility of excluding MRI from the preoperative diagnostic pathway of invasive lobular carcinoma (ILC) in women with low and low to moderate density breasts on mammography.

Methods: A total of 179 cases of ILC were diagnosed between 2009 and 2012. Forty-eight cases were identified as low and low to moderate density breasts. The study group includes 32 cases who underwent MRI. Parameters scrutinised include size and number of lesions on mammography, ultrasound and MRI, second-look ultrasound, type of surgery, further surgery and histology.

Results: Twenty-nine cases had low to moderate density breasts and three had purely low density breasts. Average age of women was 64 . Size of lesions ranged between 2 and $50 \mathrm{~mm}$ with an average of $20.14 \mathrm{~mm}$. In $25 / 32$ cases $(78.12 \%)$ conventional imaging matched MRI. MRI identified additional disease in $7 / 32(21.8 \%)$. This was predominantly in the form of satellites around the index lesion resulting in multifocality in $6 / 7$. Four resulted appropriately in mastectomy. Two led to wider WLE appropriately. In one case, multicentric disease was correctly detected and subjected to mastectomy. Second-look ultrasound was recommended in 4/7 cases. All these cases had low to moderate density breasts on mammography and 6/7 cases measured more than $15 \mathrm{~mm}$ in size. Ultrasound matched MRI in one mammographically occult case and was subjected to appropriate WLE. In two cases there was much more disease than anticipated from conventional imaging and MRI (6.25\%).

Conclusion: Even in low and low to moderate density breasts where mammography has a higher exclusion value, MRI identified additional disease in $21.8 \%(7 / 32)$.

\section{2}

Is ultrasound axillary staging less accurate in invasive lobular breast cancer than in ductal breast cancer?

P Sankaye*, G Porter, J Steel, S Doyle

Plymouth Hospitals NHS Trust, Plymouth, UK

Breast Cancer Research 2012, 14(Suppl 1):O2

Objective: To identify whether axillary US is less accurate in invasive lobular breast cancer than in ductal breast cancer.

Methods: Randomised cohorts of screening and symptomatic patients were retrospectively identified from histology records of 2010/11. Axillary US of 65 patients with primary breast cancers (BC) from each group of invasive
Table 1 (abstract 02)

\begin{tabular}{|c|c|c|c|}
\hline & IDC & ILC & $P$ value \\
\hline Node-positive BC & $34 \%(22 / 65)$ & $32 \%(21 / 65)$ & \\
\hline $\begin{array}{l}\text { Preoperative US detection of } \\
\text { node-positive disease }\end{array}$ & $54 \%(12 / 22)$ & $52 \%(11 / 21)$ & 0.5 \\
\hline Abnormal US LN appearance & $37 \%(24 / 65)^{a}$ & $26 \%(17 / 65)^{b}$ & 0.2 \\
\hline $\begin{array}{l}\text { False negative rate for axillary } \\
\text { US biopsy }\end{array}$ & $18 \%(4 / 22)$ & $12 \%(2 / 16)$ & 0.5 \\
\hline
\end{tabular}

${ }^{a}$ One unable to biopsy due to technical factors, one failure to sample node-B1. ${ }^{b}$ One failure to sample node-B1.

Table 2(abstract 02)

\begin{tabular}{lll}
\hline & IDC & ILC \\
\hline US sensitivity & $0.73(0.51$ to 0.88$)$ & $0.73(0.48$ to 0.89$)$ \\
US specificity & $0.83(0.68$ to 0.92$)$ & $0.93(0.8$ to 0.98$)$ \\
US positive predictive value & $0.70(0.48$ to 0.86$)$ & $0.82(0.55$ to 0.95$)$ \\
\hline
\end{tabular}

There were no statistically significant differences between the two groups.

lobular cancer (ILC) and invasive ductal cancer (IDC) were reviewed. Preoperative US-guided needle biopsy sampling was performed on abnormal lymph nodes (LN).

Results: See Tables 1 and 2.

Conclusion: The previous literature on this topic is inconclusive. Some authors have suggested axillary ultrasound in ILC may be less accurate than in IDC, with a higher false-negative axillary assessment rate. Another study concluded that axillary US accuracy rates in ILC were comparable with previous published studies of IDC, used FNA in all cases. We specifically compared accuracy rates of preoperative axillary staging between ILC and IDC in own institution, with $14 \mathrm{G}$ needle biopsy as the procedure of choice to sample abnormal nodes. We found that there is no statistical difference in accuracy in US axillary staging between ILC and IDC.

03

Positive predictive value of MRI vacuum biopsies in the diagnosis of nonmass-like lesions of the breast

WL Teh", EK Papantoniou, F Ng

Northwick Park Hospital, Harrow, UK

Breast Cancer Research 2012, 14(Suppl 1):O3

Objective: To evaluate the positive predictive value of MRI scoring for malignant mass-like (ML) and nonmass-like (NML) lesions based on the

\section{() Biomed Central}


BI-RADS descriptors. To identify MRI characteristics of nonmass lesions which predict malignancy for invasive and non-invasive cancers.

Methods: Retrospective analysis of $486 \mathrm{MRI}$-guided vacuum biopsies performed at Northwick Park Hospital between April 2006 and November 2011. Each lesion was categorised according to BI-RADS lexicon (ML vs. NML lesions) and time-enhancement curves, and given an overall score of MRI 1 to 5 according to overall level of suspicion for malignant disease where MRI 4 and 5 are considered suspicious or diagnostic for malignancy. Biopsy and surgical histology results obtained.

Results: A total of $291 \mathrm{ML}$ and $152 \mathrm{NML}$ lesions, of which there were 150 cancers diagnosed. Positive predictive value of MRI characteristic for malignant mass lesions is $70 \%$. Positive predictive value of MRI characteristic for nonmass lesions is $57 \%$. Segmental enhancement is the most common MRI morphology found in $45 \%$ DCIS. No specific features predict for invasive disease in NML lesions. Time-enhancement curves were mainly type $2(44.6 \%)$ and type $3(52.7 \%)$ in malignant $\mathrm{ML}$ lesions and unhelpful in predicting malignancy in NML lesions (72\% type 2 and 59\% type 3 were benign).

Conclusion: No specific BI-RADS feature predicts for invasive disease in NML lesions. Segmental enhancement is the most common MRI appearance for DCIS. Time-enhancement curves are unhelpful in predicting malignancy in NML lesions.

\section{4}

Use of MRI fusion second-look ultrasound in breast cancer: can

MRI US fusion reduce the need for MRI-guided biopsy?

SE McWilliams

Guy's and St Thomas' NHS Foundation Trust, London, UK

Breast Cancer Research 2012, 14(Suppl 1):04

Introduction: Breast MRI is being increasingly used in breast cancer to look for extent of disease, in high-risk screening and in the dense breast. Frequently incidental lesions are detected on MRI that require second-look ultrasound or stereotactic biopsy. Incidental MRI-detected lesions may be occult on conventional imaging and require MRI-guided biopsy. We describe our experiences with US MRI fusion to try and reduce the need for MRI-guided biopsy.

Methods: At our institution we have introduced an MRI breast biopsy service which is time consuming and expensive. We looked at 10 patients with MRI US fusion technology on our new Hitachi US MRI scanner to see whether lesions were easier to identify combining the MRI and US images, enabling US biopsy to be performed.

Results: Ten patients with a known breast cancer had a further incidental lesion seen on MRI. The patients had an additional supine series on contrast MRI images in addition to the standard prone protocol. The supine images were loaded on the US machine and enabled confident detection of the lesion on US in nine out of 10 patients.

Conclusion: Using MRI US fusion with one additional MRI series of supine images reduces the need for MRI-guided biopsy enabling US biopsy to be performed, which is cheaper, quicker and more patient acceptable.

\section{5}

Long-term survival of interval breast cancers in breast cancer screening in Wales

YF Fong ${ }^{1 *}$, J Evans ${ }^{2}$, D Brookes ${ }^{2}, K_{\text {K Gower Thomas }}^{1,2}$

${ }^{1}$ Royal Glamorgan Hospital, Llantrisant, UK; ${ }^{2}$ Breast Test Wales, Cardiff, UK

Breast Cancer Research 2012, 14(Suppl 1):O5

Introduction: Breast Test Wales is part of the NHS Breast Cancer Screening Programme, and oversees the screening programme in Wales. It is successful in identifying asymptomatic cancers; however, interval cancers (IC) still occur between screenings. We aim to evaluate the overall long-term survival of IC and compare that with screen-detected cancers (SDC).

Methods: Within BTW, SDC between 1998 and 2001 and IC occurring between 1998 and 2004 but screened between 1998 and 2001 were identified. IC were classified into true interval (TI), false negative (FN), occult (OCC) and unclassified (UCC). BTW receives notification of death of all women that underwent screening. The long-term survival rate was calculated from the date of initial screening and the date of death.
Results: In the 3-year screening period, 199,082 women were screened. A total of 1,020 women had SDC and 692 further developed IC following screening. Of the $692 \mathrm{IC}, 57.8 \%$ (391) were $\mathrm{TI}, 17.7 \%$ (120) were $\mathrm{FN}, 10 \%(68)$ were OCC and $14.5 \%$ were UCC. After at least 10 years of follow-up, the long-term survival rate (all-cause) for SDC was $81.6 \%$, overall for IC was $72.4 \%$ (OR 1.67, $P<0.001$ ), TI was $77.5 \%$ (OR $1.00, P=0.99$ ), FN was $55 \%$ (OR $2.36, P<0.001)$, OCC was $54.4 \%$ (OR $3.17, P<0.001)$ and UCC was $87.8 \%$ (OR $0.61, P=0.19$ ).

Conclusion: Overall survival of IC is significantly different to SDC. However SDC and TI were not statistically significantly different. FN and OCC had significantly worse long-term survival. Further research is required to identify the underlying cause of poor survival of FN and OCC.

\section{6}

Gamma probe and ultrasound-guided percutaneous localisation of the sentinel lymph node in breast cancer patients

P Whelehan*, A Evans, S Vinnicombe, D Brown, D McLean

University of Dundee, UK

Breast Cancer Research 2012, 14(Suppl 1):O6

Introduction: A major reason for failure to diagnose axillary lymph node metastasis preoperatively in breast cancer patients is that needle biopsy may not target the sentinel node (SLN). We aimed to address this by testing the accuracy with which we could identify and target the sentinel lymph node percutaneously under combined radioisotope and ultrasound guidance.

Methods: Ethical approval was obtained. In 48 patients scheduled for surgical sentinel lymph node biopsy (SLNB), following injection of radioisotope, one of three radiologists used a gamma probe in tandem with ultrasound guidance to identify a SLN prior to surgery and mark it with a localising wire. The patients then proceeded to surgical SLNB, guided by radioisotope and blue dye. The surgeon noted whether the wire had correctly marked a SLN.

Results: The SLN was correctly localised in $75 \%$ of patients ( 36 of $48 ; 95 \%$ $\mathrm{Cl}=63$ to $87 \%$ ). A learning curve for the three operators was observed, with a rate of correct localisation in the first five procedures performed by each operator of $67 \%$ (10 of 15) rising to $79 \%$ in the subsequent 33 (26 of $33 ; 95 \% \mathrm{Cl}=64$ to $93 \%$ ). Interoperator variations in accuracy were evident, with a success rate of $83 \%$ in the most experienced of the three.

Conclusion: Percutaneous SLN localisation using combined radioisotope and ultrasound guidance is feasible. Use of this method to guide needle biopsy of the axilla could increase the preoperative diagnosis rate for axillary lymph node metastases in breast cancer patients.

\section{POSTER PRESENTATIONS}

P1

Abstracts P1 - P16 are educational and not included for publication online

Breast Cancer Research 2012, 14(Suppl 1):P1

Abstracts P1 - P16 are educational and not included for publication online

P2

Abstracts P1 - P16 are educational and not included for publication online

Breast Cancer Research 2012, 14(Suppl 1):P2

Abstracts P1 - P16 are educational and not included for publication online

P3

Abstracts P1 - P16 are educational and not included for publication online

Breast Cancer Research 2012, 14(Suppl 1):P3

Abstracts P1 - P16 are educational and not included for publication online 
P4

Abstracts P1 - P16 are educational and not included for publication online

Breast Cancer Research 2012, 14(Suppl 1):P4

Abstracts P1 - P16 are educational and not included for publication online

P5

Abstracts P1 - P16 are educational and not included for publication online

Breast Cancer Research 2012, 14(Suppl 1):P5

Abstracts P1 - P16 are educational and not included for publication online

P6

Abstracts P1 - P16 are educational and not included for publication online

Breast Cancer Research 2012, 14(Suppl 1):P6

Abstracts P1 - P16 are educational and not included for publication online

P7

Abstracts P1 - P16 are educational and not included for publication online

Breast Cancer Research 2012, 14(Suppl 1):P7

Abstracts P1 - P16 are educational and not included for publication online

P8

Abstracts P1 - P16 are educational and not included for publication online

Breast Cancer Research 2012, 14(Suppl 1):P8

Abstracts P1 - P16 are educational and not included for publication online

P9

Abstracts P1 - P16 are educational and not included for publication online

Breast Cancer Research 2012, 14(Suppl 1):P9

Abstracts P1 - P16 are educational and not included for publication online

P10

Abstracts P1 - P16 are educational and not included for publication online

Breast Cancer Research 2012, 14(Suppl 1):P10

Abstracts P1 - P16 are educational and not included for publication online

P11

Abstracts P1 - P16 are educational and not included for publication online

Breast Cancer Research 2012, 14(Suppl 1):P11

Abstracts P1 - P16 are educational and not included for publication online
P12

Abstracts P1 - P16 are educational and not included for publication online

Breast Cancer Research 2012, 14(Suppl 1):P12

Abstracts P1 - P16 are educational and not included for publication online

P13

Abstracts P1 - P16 are educational and not included for publication online

Breast Cancer Research 2012, 14(Suppl 1):P13

Abstracts P1 - P16 are educational and not included for publication online

P14

Abstracts P1 - P16 are educational and not included for publication online

Breast Cancer Research 2012, 14(Suppl 1):P14

Abstracts P1 - P16 are educational and not included for publication online

P15

Abstracts P1 - P16 are educational and not included for publication online

Breast Cancer Research 2012, 14(Suppl 1):P15

Abstracts P1 - P16 are educational and not included for publication online

P16

Abstracts P1 - P16 are educational and not included for publication online

Breast Cancer Research 2012, 14(Suppl 1):P16

Abstracts P1 - P16 are educational and not included for publication online

P17

Breast density measurement for personalised screening

JC Sergeant ${ }^{1 *}$, S Musa $^{2}$, M Wilson $^{3}$, DG Evans ${ }^{3}$, A Howell $^{3}$, SM Astley

${ }^{1}$ Institute of Population Health, University of Manchester, UK;

${ }^{2}$ Manchester Medical School, University of Manchester, UK;

${ }^{3}$ Nightingale Centre and Genesis Prevention Centre, University Hospital of

South Manchester, Manchester, UK

Breast Cancer Research 2012, 14(Suppl 1):P17

Introduction: Breast density is both a modifiable risk factor for breast cancer and an indicator of the sensitivity of mammography. Reliable measurement in the screening population could enable personalised screening to maximise early detection of cancer. In the Predicting Risk of Cancer at Screening (PROCAS) study, we are investigating different approaches to measuring breast density, and we present comparative data from one subjective method and two automated volumetric methods.

Methods: The screening mammograms of 4,109 women enrolled in PROCAS were visually assessed independently by two experienced film readers, who recorded their estimates of percentage density on visual analogue scales (VAS). The mammograms were also processed by Quantra ${ }^{\text {TM }}$ (Hologic Inc.) and Volpara (Matakina Technology Ltd). They were ranked according to density by each method, and the top $10 \%$ and $1 \%$ compared. Results: Of the 617 mammograms ranked in the most dense $10 \%$ by at least one method, only 127 were high density by all three methods. The overlap between the two volumetric methods was 214; between VAS and Quantra $^{\mathrm{TM}}$ it was 195 and between VAS and Volpara it was 147. For the 
48 mammograms in the top $1 \%$ by density, the overlaps were 13,9 and 7 , respectively.

Conclusion: The lack of overlap between the methods was surprising and has serious implications for the implementation of personalised screening. The optimum measure of breast density in digital mammograms has yet to be identified; different methods may yield the strongest links with cancer risk and sensitivity of mammography, and further research is needed to elucidate these relationships.

\section{P18}

Changes in recall rate, biopsy rate and cancer detection since the introduction of digital mammography

TG Fyall", CRM Boggis, SM Astley, JC Sergeant, J Morris, S Whiteside, M Wilson The University of Manchester, UK

Breast Cancer Research 2012, 14(Suppl 1):P18

Introduction: Analogue mammography is gradually being replaced by digital mammography for breast screening. Digital mammography has been shown to be at least as good as analogue mammography at cancer detection, but the picture regarding other outcomes such as recall rate and biopsy rate is less clear.

Methods: Outcome data from 62,599 routine screening mammograms taken in Greater Manchester between January 2010 and March 2012 were gathered from screening records. Mean recall, biopsy/cytology and cancer detection rates were calculated for analogue and digital screening and compared. After complete conversion to digital screening, the same three measures were obtained on a monthly basis over 2 years. The data were analysed to identify any trends in the measures.

Results: The digital mammography recall rate was $4.69 \%(2,505 / 53,444)$, with analogue significantly lower at $3.58 \%(328 / 9,155)(P<0.001)$. The digital biopsy/cytology rate was $1.88 \%(1,005 / 53,444)$, with analogue significantly lower at $1.49 \%(136 / 9,155)(P=0.01)$. The cancer detection rate (per 1,000 screens) was $6.74(56 / 9,155)$ for digital mammography and 6.15 $(358 / 53,444)$ for analogue; however, this difference was not significant $(P=$ $0.53)$. The recall rate showed a strong positive correlation with time $(r=0.71$, $P<0.001)$ and the biopsy rate showed a weak positive correlation $(r=0.52$ $P=0.011$ ). Cancer detection showed a weak negative correlation with time $(r=-0.567 P=0.05)$.

Conclusion: The introduction of digital mammography resulted in higher recall and biopsy rates, but no change in cancer detection. Recall and biopsy rates, whilst still well within recommended levels, appear to be increasing since the introduction of digital mammography; this indicates a need for regular monitoring.

P19

The Nottingham Prognostic Index: 5-year and 10-year survival data for all-cause survival within a screened population

YF Fong ${ }^{1 *}$, J Evans ${ }^{2}$, D Brookes ${ }^{2}$, K Gower Thomas ${ }^{1,2}$

${ }^{1}$ Royal Glamorgan Hospital, Llantrisant, UK; ${ }^{2}$ Breast Test Wales, Cardiff, UK Breast Cancer Research 2012, 14(Suppl 1):P19

Introduction: The Nottingham Prognostic Index (NPI) is an accepted prognostication tool in the management of breast cancers. The latest overall 5-year and 10-year breast cancer survival has been reported to be $85 \%$ [1] and $77 \%$ [2]. We applied the NPI to breast cancers diagnosed within Breast Test Wales and present survival data in each NPI category.

Methods: All women with screen-detected and interval cancers having had screening between 1998 and 2001 were included. The NPI for each cancer was calculated with the size, nodal status and grade of the primary tumour. Survival data (all cause) was calculated after 10 years of follow-up.
Results: In the 3-year screening period, 199,082 women were screened. A total of 1,712 cancers were diagnosed. In total, 1,546 had data available for calculating the NPI. The overall 5-year and 10-year survival was $94 \%$ and $82 \%$. See Table 1.

Conclusion: The overall 5-year and 10-year survival (all cause) has improved even when compared with specific breast cancer survival of recent published data. Our data provide a reference for updating all-cause survival of women diagnosed with breast cancers within a screened population. References

1. Office for National Statistics: Statistical Bulletin: Cancer Survival in England: Patients Diagnosed 2005-2009 and Followed up to 2010-2011 London: Office for National Statistics 2011.

2. Coleman MP, et al: Cancer survival in Australia, Canada, Denmark, Norway, Sweden, and the UK, 1995-2007 (the International Cancer Benchmarking Partnership): an analysis of population-based cancer registry data. The Lancet 2010, 377:127-138.

P20

Impact of full-field digital mammography on diagnostic work-up and surgical management of mammographic microcalcification

SM Bundred ${ }^{1 *}$, J Zhou ${ }^{1}$, S Whiteside ${ }^{1}$, J Morris $^{2}$, NJ Bundred ${ }^{2}$

${ }^{1}$ University Hospital South Manchester, Manchester, UK; ${ }^{2}$ University of Manchester, UK

Breast Cancer Research 2012, 14(Suppl 1):P20

Introduction: Full-field digital mammography (FFDM) increases detection of benign and malignant calcified breast lesions. Accurate nonoperative diagnosis of malignant impalpable breast lesions minimises numbers of therapeutic surgical procedures. Correct diagnosis of malignant mammographic microcalcification (MM) is important because upgraded lesions require repeat surgical procedures in 57\% of cases (NHSBSP Audit 2012).

Methods: Screening and symptomatic women with $\mathrm{MM}(n=1,479)$ were reviewed to determine the impact of FFDM (imaging with FFDM only since April 2010) on the positive predictive value (PPV), diagnostic accuracy and surgical management of MM. Demographic information, preoperative and postoperative diagnosis and number of surgical procedures were recorded for Group 1 (August 2007 to March 2010: $n=711$ ) and Group 2 (April 2010 to May 2011: $n=768$ )

Results: Reduction in PPV of biopsy for MM was observed (Group 1, 42.6\%: Group 2, 32.7\%; $P<0.0001)$. Correct or concordant nonoperative diagnosis increased with FFDM (Group 1, 89\% vs. Group 2, 95\%; $P<0.0001$ ) and was achieved more often at first attempt (Group 1, 80.6\% vs. Group 2, 89.5\%; $P<$ 0.0001). More lesions under $5 \mathrm{~mm}$ were biopsied using FFDM (Group 1, 15\%; Group 2, 20.4\%; $P=0.008$ ). Accurate preoperative diagnosis of malignancy permitted single-stage surgery in $77.4 \%$ Group 2 versus $67.9 \%$ Group 1 ( $P=$ 0.017). For DCIS cases, similar first-line mastectomy rates were observed (Group 1, 30.3\% vs. Group 2, 33.3\%, $P=\mathrm{NS}$ ). Fewer B3/4 lesions upgraded at surgery (Group 1, 48.7\% vs. Group 2, 20\%; $P=0.011$ ).

Conclusion: Nonoperative work-up of MM using FFDM reduced second therapeutic procedures for MM, decreased upgrade of B3/4 lesions at diagnostic surgery, but increased benign nonoperative biopsies for MM.

P21

Abstract withdrawn by author

Breast Cancer Research 2012, 14(Suppl 1):P21

Abstract withdrawn by author:

\section{Table 1(abstract P19)}

\begin{tabular}{llll}
\hline NPI category & Number of cases & 5-year survival (\%) & 10-year survival (\%) \\
\hline 1 & $322(20.8 \%)$ & 97 & 89 \\
2 & $537(34.7 \%)$ & 93 & 84 \\
3 & $536(34.7 \%)$ & 90 & 77 \\
4 & $151(9.8 \%)$ & 88 & 73 \\
\hline
\end{tabular}


P22

3T MR imaging: diffusion-weighted and dynamic contrast-enhanced relationship of apparent diffusion coefficient value and maximum percentage enhancement in invasive lobular carcinoma of the breast ID Lyburn ${ }^{1,2^{*}}$, HC Russell $^{1,2}$, J Searle ${ }^{2}$, C Croucher $^{1,2}$, DO Hall ${ }^{2}$

${ }^{1}$ Thirlestaine Breast Centre, Cheltenham, UK; ${ }^{2}$ Cheltenham Imaging Centre, Cheltenham, UK

Breast Cancer Research 2012, 14(Suppl 1):P22

Introduction: In breast MR imaging, diffusion-weighted imaging (DWI) and dynamic contrast-enhanced (DCE) studies are being increasingly utilised in assessing malignancy. We investigated potential relationships between these two techniques in cases of proven invasive lobular carcinoma (ILC).

Methods: Thirty-two consecutive cases of ILC underwent 3T breast MR imaging as part of routine staging - retrospective analysis was undertaken. Diffusion-weighted imaging using three $b$ values $(0,500$ and 1,000 seconds $/ \mathrm{mm}^{2}$ ) was performed prior to dynamic contrast-enhanced imaging in each case. Post processing included obtaining maximum intensity projections and multiplanar reconstruction. Regions of interest (ROI) were placed over the index (presenting) lesion. In cases of multifocality/multicentricity, the most conspicuous enhancing lesion was interrogated. Enhancement kinetics were evaluated from the dynamic contrast-enhanced images: the percentage of signal intensity increase within the first 2 minutes after administrating contrast agent relative to pre-contrast signal intensity was calculated and the morphology of the curve for 6 minutes after contrast administration was analysed. The same size of ROI was placed over the lesion site on the DWI and the apparent diffusion coefficient (ADC) map was calculated.

Results: Age range, 34 to 74 years; index lesion size, 4 to $78 \mathrm{~mm}$. For type 2 and type 3 curves, signal increase was 128 to 306\%; ADC mean $1.29 \times 10^{-3} \mathrm{~mm}^{2} / \mathrm{second}$. In type 1 curves, signal increase was 82 to $93 \%$; ADC mean $0.85 \times 10^{-3} \mathrm{~mm}^{2} /$ second. In four cases (with type 1 or type 2 curves) no lesions with restricted diffusion were apparent.

Conclusion: The percentage signal intensity increase on DCE is higher in cases with measurable ADC values than without. ILC with type 1 or type 2 curves may not be visible on DWI.

P23

Abstract withdrawn

Breast Cancer Research 2012, 14(Suppl 1):P23

\section{P24}

Fatigue and performance in interpreting breast screening mammograms

S Taylor-Phillips ${ }^{1 *}$, A Clarke $^{1}$, M Wheaton ${ }^{2}$, O Kearins ${ }^{3}$, M Wallis $^{4}$

${ }^{1}$ The University of Warwick, Coventry, UK; ${ }^{2}$ University Hospital Coventry and

Warwickshire, Coventry, UK; ${ }^{3}$ West Midlands Quality Assurance Reference

Centre, Birmingham, UK; ${ }^{4}$ Addenbrookes Hospital, Cambridge, UK

Breast Cancer Research 2012, 14(Suppl 1):P24

Introduction: Interpreting mammograms is a repetitive visual task, which may cause fatigue. Current practice in England for digital mammography is that both readers examine batches of mammograms in the same order as one another. This research examines whether there is a potential benefit in the two readers examining the cases in a different order to one another, to ameliorate any effects of fatigue at the system level.

Methods: NBSS records at one screening centre for 4 years were examined (2007 to 2010 , seven readers, $>170,000$ cases). The time and date that each case was reported was extracted from NBSS. A period of over 1 hour without reporting any cases was defined as a break. Recall rate was compared for the first 10 cases since a break and the 10 cases after that using a within-subjects $t$ test.

Results: Each reader examined between 20,080 and 74,028 cases over 4 years, and recall rates ranged from $3.6 \%$ to $5.9 \%$. Recall rate was $2.3 \%$ higher for the first 10 cases after the break than for the subsequent 10 cases $(P=0.004)$. The sample was too small to examine effects on cancer detection rate.
Conclusion: There may be patterns in performance with time since a break. Further research is needed to ascertain whether these patterns remain present in a larger more controlled sample, and whether changing case order could improve overall performance. The Changing case Order to Optimise patterns of Performance in Screening (CO-OPS) randomised controlled trial will be begin recruitment in England soon to address these questions.

P25

Role of targeted reassurance breast ultrasound in women with symptoms of a breast lump and normal clinical examination R Gallagher*, H Burrell, E Cornford

Nottingham Breast Institute, Nottingham, UK

Breast Cancer Research 2012, 14(Suppl 1):P25

Introduction: Ultrasound has a well-established role in the triple assessment of breast lumps. The purpose of this study was to review the value of targeted breast ultrasound in women presenting with symptoms of a breast lump but normal clinical examination.

Methods: The records of all patients presenting to the symptomatic clinic with a breast lump but with a normal clinical examination over a 6-month period were reviewed. In our institution all such patients undergo targeted ultrasound of the symptomatic area within the breast; and mammography if aged 40 years or over. The results of the ultrasound examination, mammography and histology from any needle biopsy were reviewed.

Results: Seven hundred and ninety-nine women were included (mean age = 39 , age range 15 to 84 ). One hundred and two (13\%) had an abnormality detected on ultrasound, $92(90 \%)$ of which were at the same site as the patient's symptoms. Thirty-two patients ( $4 \%$ of the study group) had solid lumps and $70(9 \%)$ had cysts. Five cancers were detected, four of which were at the site of the patient's symptoms and one of which was an incidental area of DCIS visible on mammography only. Three of the four patients with invasive cancer had a normal mammogram.

Conclusion: Ultrasound is a useful diagnostic tool in patients who present with a breast lump, but who have a normal clinical examination. In this study a small number of cancers were detected. The value of a normal or benign ultrasound in reassuring both patient and clinician is more difficult to quantify.

P26

A comparison of reading times in full-field digital mammography and digital breast tomosynthesis

SJ Connor ${ }^{* *}$, YY Lim² ${ }^{2}$ C Tate ${ }^{3}$, H Entwistle ${ }^{2}$, J Morris ${ }^{4}$, S Whiteside ${ }^{4}$

J Sergeant ${ }^{3}$, M Wilson ${ }^{2}$, U Beetles ${ }^{2}$, C Boggis ${ }^{2}$, F Gilbert ${ }^{5}$, S Astley ${ }^{3}$

${ }^{1}$ Manchester Medical School, University of Manchester, UK; ${ }^{2}$ Nightingale

Breast Centre, University Hospital of South Manchester, Manchester, UK;

${ }^{3}$ Institute of Population Health, University of Manchester, UK; ${ }^{4}$ Department of Medical Statistics, University of Manchester, UK; ${ }^{5}$ School of Clinical Medicine, University of Cambridge, UK

Breast Cancer Research 2012, 14(Suppl 1):P26

Introduction: Currently, breast screening is implemented with X-ray mammography, but new technology such as digital breast tomosynthesis (DBT) may provide significant advantages because it produces threedimensional breast images with high spatial resolution. To aid assessment of the cost-effectiveness of DBT, we have compared reading times for DBT images and full-field digital mammograms (FFDM) of the same women.

Methods: Four consultant radiologists were timed by stopwatch reading FFDM images and then DBT images, with a total of 119 timings of two view cases for each imaging modality. The mammograms used were from women recalled following a screening mammogram and from women who are screened annually due to a family history of breast cancer. The time taken to display the images and to report on them was excluded from the analysis.

Results: The reading time was significantly longer for DBT images than for FFDM images, with median times of 66 seconds and 17 seconds, respectively $(P<0.001)$, with times for FFDM ranging from 4.7 to 109.0 seconds and for DBT from 31.9 seconds to 180.6 seconds. The ratio of reading times DBT: FFDM was approximately $4: 1$, with readers varying between ratios of 2.4 to 4.9 . 
Conclusion: Reading times were greater for DBT as expected, since the reader has to scroll through the images slices to visualise the entire breast. The images used in this study were likely to have a higher rate of abnormalities than screening mammograms, and to have higher breast density, both of which will increase reading time over routine screening.

P27

Repeatability of breast density visual assessment

L Walshaw ${ }^{1 *}$, JC Sergeant ${ }^{2}$, M Wilson ${ }^{3}$, S Steed ${ }^{1}$, N Barr $^{3}$, U Beetles ${ }^{3}$, C Boggis $^{3}$,

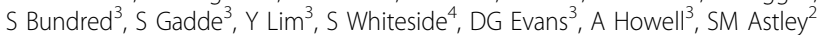
${ }^{1}$ Manchester Medical School, University of Manchester, UK; ${ }^{2}$ Institute of Population Health, University of Manchester, UK; ${ }^{3}$ Nightingale Centre and Genesis Prevention Centre, University Hospital of South Manchester, Manchester, UK; ${ }^{4}$ Department of Medical Statistics, University Hospital of South Manchester, Manchester, UK

Breast Cancer Research 2012, 14(Suppl 1):P27

Introduction: Breast density, measured as the proportion of the breast occupied by fibroglandular tissue in a mammogram, is a strong and modifiable risk factor for breast cancer. Area-based estimates made by expert observers are a practical approach, but are subjective. Here we investigate repeatability of visual assessment of percentage breast density.

Methods: Seven mammographic film readers re-assessed the density of 100 normal full-field digital mammogram cases for which they had made density estimates at least 1 year previously as part of the Predicting Risk of Cancer at Screening (PROCAS) study. The mammograms for a given reader were selected to show a range in density, by randomly sampling 10 cases from each decile of density assessed by that reader. They were reviewed in similar reading conditions on both occasions using a visual analogue scale to record the assessments.

Results: For the majority of readers the difference in mean density between the two sets of readings was less than $6 \%$, but the largest discrepancy between means was $14.7 \%$. Bland-Altman plots were generated for each reader and showed considerable variation between readings on the two occasions. At best, the limits of agreement were $-12.46 \%$ to $+17.02 \%$, and at worst they were $-14.50 \%$ to $+40.98 \%$. The largest difference between first and second readings for each reader ranged from 26 to $65 \%$.

Conclusion: Although density estimates made by a subset of these readers have been strongly related to cancer risk, the variability in reproducibility calls into question the usefulness of subjective assessment without prior evaluation of reader performance.

\section{P28}

Structured evaluation of the effectiveness of an interactive tool for developing interpretation skills in mammography

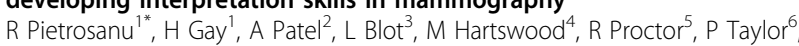
L Wilkinson

'St Georges Hospital NHS Trust, London, UK; ${ }^{2}$ The Royal Surrey County Hospital NHS Foundation Trust, Guildford, UK; ${ }^{3}$ University of York, UK;

${ }^{4}$ University of Edinburgh, UK; ${ }^{5}$ University of Middlesex, UK;

${ }^{6}$ University College London, UK

Breast Cancer Research 2012, 14(Suppl 1):P28

Introduction: Lesion Zoo, a computer-based training tool, was developed to give trainee radiologists access to a wide range of possible mammographic appearances. Users describe lesions using terms from the BI-RADS lexicon and are given feedback on how their descriptions compare with those of by a panel of experts.

Methods: A zoo is a collection of lesions presented to a trainee, who is invited to ascribe features to the lesions and provide a confidence estimate. Trainees receive feedback on their progress, through a detailed comparison of their assessment with that of a panel of experts, and from a learning curve showing how their confidence changes. During this evaluation, two sets of test cases (masses and calcifications) were offered initially as a baseline assessment, followed by training on masses or calcifications, followed by a second assessment after which training was swapped (the group that had initially trained on masses was trained on calcifications and vice versa), and then a third assessment was carried out, allowing a test of the impact of the training on performance.
Results: Analysis of variance shows significant two-way interaction: participants receiving training on calcifications improve more on calcifications than on masses and participants receiving training on masses improve more on masses.

Conclusion: Computer-based training tools provide a valuable addition to conventional training and allow trainees to get rapid access to experience with large numbers of cases. Well-designed tools that provide meaningful feedback on interpretation tasks are likely to be effective in improving performance.

\section{P29}

Contrast-enhanced MRI in evaluating the contralateral breast

for synchronous malignancy in patients with invasive lobular cancer

R Pietrosanu*, MA Shah, H Gay, S Heller, M Reddy

St Georges Hospital NHS Trust, London, UK

Breast Cancer Research 2012, 14(Suppl 1):P29

Aim: To evaluate the use of MRI in assessment of the contralateral breast in women with invasive lobular cancer.

Methods: Retrospective review of 157 breast MRIs in our local unit from January 2010 to May 2012 was carried out. Of these, 35 women had biopsyproven invasive lobular cancer. Dotarem-enhanced 1.5T MRI was performed and UK standard reporting criteria were used to grade suspicion in any contralateral lesions alongside the use of contrast enhancement curves.

Results: Bilateral MRI demonstrated nine (24\%) contralateral lesions graded as MRI 2 or above, warranting further investigation with secondlook ultrasound with five proceeding to ultrasound core biopsy. Of these, three were confirmed as malignant, revealing three contralateral invasive lobular cancers and one low-grade ductal carcinoma in situ. Our results demonstrated contralateral malignancy in $8 \%$ of women with invasive lobular disease.

Conclusion: Current UK guidance does not routinely recommend MRI screening of the contralateral breast in patients with invasive lobular carcinoma. Studies have shown there is a wide variation in detection of contralateral malignancy from 3 to $18 \%$. Our review showed a relatively high rate of incidental contralateral malignancy of $8 \%$, suggesting that MRI screening of the contralateral breast may have more of a role in this patient group than initially considered. We are now extending this review to include three other hospitals in South West London Breast Cancer Network to evaluate these initial findings further.

\section{P30}

Imaging the breast following neoadjuvant chemotherapy: does improvement of imaging findings in the axilla predict pathological response in the breast?

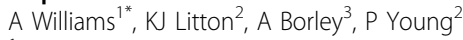

${ }^{1}$ School of Medicine, Cardiff University, Cardiff, UK; ${ }^{2}$ Cardiff and Vale Breast Centre, University Hospital Llandough, Cardiff, UK; ${ }^{3}$ Velindre Hospital, Velindre NHS Trust, Cardiff, UK

Breast Cancer Research 2012, 14(Suppl 1):P30

Introduction: Neoadjuvant chemotherapy is an effective treatment in patients with locally advanced breast cancer. It is being used more frequently and imaging is used to monitor disease response. Imaging response in the axilla was correlated with the final breast histology.

Methods: Patients who had undergone primary chemotherapy, pre and post chemotherapy MRI and surgery from 2009 to present were included. The MRI and histology reports were reviewed.

Results: Of 20 patients, 18 had abnormal nodes on the first MRl; of these nine had a complete imaging response and five had axillary disease on final pathology. Of the nine that had abnormal nodes on their final MRI, all but one had metastatic disease on final pathology. Of the nine patients with a response on axillary imaging, two had a complete pathological response in the breast. The other had an average tumour size of $53 \mathrm{~mm}$ (range 16 to $120 \mathrm{~mm}$ ). Of the patients who did not respond radiologically in the axilla, three had a complete pathological response in the breast and the remainder had an average tumour size of $44 \mathrm{~mm}$ (range 1.7 to $90 \mathrm{~mm}$ ).

Conclusion: Although MRI is used to predict response to chemotherapy it may underestimate and overestimate disease burden. A radiological response in the axilla does not predict a response in the breast and the 
two areas need to be assessed independently of each other. Surgical planning must take into consideration the pre-chemotherapy disease status in each area.

\section{P31}

Does imaging correctly influence the surgical management in the setting of neoadjuvant chemotherapy?

H Fatayer*, B Kim, N Sharma, DD Manuel, BJG Dall

Leeds Teaching Hospitals NHS Trust, Leeds, UK

Breast Cancer Research 2012, 14(Suppl 1):P31

Objective: To evaluate the role of imaging in aiding surgical management and assess whether imaging resulted in unnecessary mastectomies.

Methods: A retrospective analysis of 165 patients with invasive breast cancer treated with neoadjuvant chemotherapy (NAC) (2005 to 2009). Bilateral cancers were analysed separately (total $=169$ ). Data collected include tumour extent on mammogram and MRI, radiology advice for surgery, surgery performed, and whole tumour size on postsurgical specimens. MRI report recommended wide-local excision (WLE), mastectomy, or WLE with multidisciplinary team discussion (MDTD) based on imaging. We used residual tumour size $<40 \mathrm{~mm}$ on pathology as a cut-off for suitability of WLE.

Results: Radiology advice regarding surgery was suggested in 167 cases and advice was followed in $142(85 \%)$ cases. Fifty-nine cases were suggested for WLE; 46 (78\%) underwent WLE with six requiring further surgery (no further disease in four) and $13(22 \%)$ underwent mastectomy based on patient's choice or clinical decision. Ninety-one cases were suggested for mastectomy; two (2\%) had successful WLE due to patient's choice (one had therapeutic mammoplasty) and 89 (98\%) had mastectomy. Seventy-four were appropriate (inflammatory, multicentric, pathology $\geq 40 \mathrm{~mm}$, patient choice or surgical cosmesis) and based on pathology 15 may have had inappropriate mastectomies as residual disease $<40 \mathrm{~mm}$. Seventeen cases were considered for WLE with MDTD, seven (41\%) had successful WLE and 10 (59\%) had mastectomy; all were appropriately advised when compared with final pathology.

Conclusion: This series suggests that NAC offers more opportunities for breast conservation than are being realised. Multifocal disease and extensive calcifications may not mandate mastectomy if the patient responds well to chemotherapy.

P32

\section{Film-reading workload in the West Midlands}

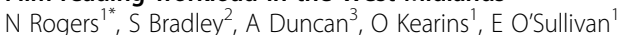

'West Midlands Breast Screening Quality Assurance Reference Centre,

Birmingham, UK; ${ }^{2}$ South Birmingham Breast Screening Service, Birmingham, UK;

${ }^{3}$ Warwickshire, Solihull \& Coventry Breast Screening Service, Coventry, UK

Breast Cancer Research 2012, 14(Suppl 1):P32

Introduction: The NHS Breast Screening Programme (NHSBSP) stipulates that film-readers should report a minimum of 5,000 cases per year. However, there is no stipulated maximum caseload. With the increasing number of women being included in the remit of the NHSBSP, the question of a maximum is becoming more relevant.

Methods: Over the period 1 April 2008 to 31 March 2011 there were 37 radiologists and 34 advanced practitioners reporting breast screening cases within the West Midlands BSP. The range of screening cases reported during this period was between 35 and 49,053 per reader. Readers were divided into four groups according to total breast screening cases they had reported over the 3-year period $(<15,000,15,000$ to $20,000,20,000$ to 25,000 and $>25,000$ ), in order to assess film-reader performance by number of cases reported. Only those who reported in the West Midlands for the full 3-year period were included $(n=47)$. This was to enable direct comparisons with Cornford and colleagues, who reported that performance in readers reporting $>25,000$ cases (over a 3-year period) may decline [1].

Results: The group reading 20,000 to 25,000 cases had a significantly lower recall rate than the other three groups $(P<0.01)$ and a higher PPV $(P<0.01)$. The missed cancer rate and cancer detection rate showed no difference.

Conclusion: The findings are not in agreement with previously published data suggesting that these parameters should not be applied as a quality standard to the NHSBSP. Performance will be continually monitored within the West Midlands over the coming years.

Reference

1. Cornford, et al: Clin Radiol 2011, 66:103-107.

P33

Abstract withdrawn

Breast Cancer Research 2012, 14(Suppl 1):P33

\section{P34}

Clinical recall from the NHS Breast Screening Programme: is it worth doing?

M Morgan*, J Helsdon, A Thomson

Dorset Breast Screening Unit, Poole, UK

Breast Cancer Research 2012, 14(Suppl 1):P34

Introduction: Within the NHSBSP, ladies with normal mammograms may be recalled, if they proffer significant clinical symptoms. A clinical recall may then be initiated by the reader according to local guidelines. The purpose of this study was to determine the recall rate of ladies undergoing clinical recall and the cancer detection rate in this group.

Methods: The entire South West and South Central Health Authority screening population was examined for 2011. The study analysed the number of ladies undergoing breast screening, the number of ladies proceeding to clinical recall and the number of cancers detected following clinical recall.

Results: A total of 342,628 women were screened within these two regions in 2011. Of these ladies, 884 were recalled for a clinical recall. This represents an overall clinical recall rate of $0.26 \%$ or one clinical recall for every 388 patients screened. Of the 884 patients recalled, 17 of these patients were subsequently diagnosed with breast cancer. This represents one breast cancer detected for every 52 women clinically recalled to assessment.

Conclusion: Clinical recall does detect breast cancer but 52 women must be recalled to detect one cancer. These ladies are included in the recall rate of the NHSBSP, but the cancer detection rate in this subset of patients is relatively poor compared with those recalled with abnormal mammography. The NHSBSP should evaluate whether this is an acceptable detection rate or use of resources. An alternative is that these ladies could be directed to their $\mathrm{GP}$, and referred onwards to the symptomatic breast service, when clinically appropriate.

P35

Annual screening of moderate-risk women: a review of 10 years

experience within the NHS Breast Screening Programme

ND Forester*, CE Holmes, N Sibal, L McLean

Department of Breast Radiology, Newcastle upon Tyne, UK

Breast Cancer Research 2012, 14(Suppl 1):P35

Introduction: We have offered annual breast screening to women under 50 years with moderate risk of breast cancer (as defined by NICE guidelines) within the NHS Breast Screening Programme in Newcastle for over 10 years. We evaluated the process and outcome of this programme in this subpopulation of screened women.

Methods: A retrospective review of women screened between April 2002 and March 2012. The population screened, screening episodes, recall rate and outcomes were assessed.

Results: A total of 1,027 moderate risk women were screened over 10 years, resulting in 5,406 screening episodes generated. There were 135 recalls from screening in 126 women, giving a recall rate of $2.5 \%$. Of the 135 recall episodes, 100 were benign on further imaging and clinical examination. Thirty-five underwent biopsy, of which 13 were malignant. This gives a cancer detection rate of 2.4 per 1,000 women screened.

Conclusion: Screening of moderate-risk women is possible within the NHS Breast Screening Programme. The recall rate of $2.5 \%$ falls within the acceptable standards for both prevalent and incident screens set in the over-50 years population. As this is annual screening, the overall cancer 
detection rate is similar to that found in the over- 50 years group (approximately 6 per 1,000 over 3 years). Screening is therefore as effective in this increased risk population as the current NHS Breast Screening programme is for over-50 year olds.

Reference

1. National Collaborating Centre for Primary Care: Familial Breast Cancer: The Classification and Care of Women At Risk of Familial Breast Cancer in Primary, Secondary and Tertiary Care London: NICE 2006, 1-75.

\section{P36}

Survey of clinical recalls from breast screening at Nottingham Breast Institute: implications for practice

MZ Mvere*, S Tennant

Nottingham Breast Institute, Nottingham, UK

Breast Cancer Research 2012, 14(Suppl 1):P36

Introduction: In our unit, symptomatic women identified at the time of screening (that is, those reporting a breast symptom, or in whom the radiographer notices a breast sign) are recalled for further assessment despite a normal mammogram. Several studies have suggested that the cancer detection rate in this group of patients is low. The aims of this survey were therefore to determine the cancer yield and whether our current practice of recalling women on the basis of clinical history alone is a worthwhile practice.

Methods: The total number of women screened, and those recalled to assessment, during a 24-month period were retrospectively identified using NBSS. Women recalled on the basis of clinical history alone were identified and information about the screening assessment clinic visit was reviewed.

Results: Of the 45,940 women screened during this time period, 337 cancers (invasive and DCIS) were diagnosed ( 7.3 cancers/1,000 women screened). A total of 335 cancers were diagnosed in the 1,177 women recalled because of an abnormal mammogram (28.4\%). Two cancers were detected in the 116 women recalled for clinical history alone $(1.72 \%)$. The cancer detection rate is therefore 17 times lower in the clinical recall group than in those called for a mammographic abnormality.

Conclusion: The cancer detection rate in women recalled on the basis of clinical history alone is low and, given the extra time and resources required in assessing these women, our current practice should be reviewed.

P37

\section{Imaging features of noncalcified DCIS}

JM Scudder, J Parikh

Guy's and St Thomas' NHS Foundation Trust, London, UK

Breast Cancer Research 2012, 14(Suppl 1):P37

Objective: To illustrate the radiological features of noncalcified DCIS (NCDCIS) on mammography, ultrasound and MRI. To highlight the role of MRI in determining extent of disease.

Introduction: DCIS accounts for $20 \%$ screen-detected cancers and $5 \%$ of symptomatic cancers. Eighty to $90 \%$ present mammographically as microcalcifications, but 10 to $20 \%$ are noncalcified and can be mammographically occult. With reported re-excision rates as high as $65 \%$ for breast-conserving surgery in DCIS, accurately determining disease extent on preoperative imaging is important.

Methods: Imaging of 117 patients with pure DCIS from 2007 to 2011 was reviewed retrospectively. Fifteen patients with NC-DCIS were identified. Imaging findings were compared with disease extent on postoperative histology.

Results: NC-DCIS appeared as follows. Mammography: occult 33\%, diffuse increased breast density 33\%, focal architectural distortion 13\%, wellcircumscribed lesion $13 \%$, tubular ductal density $7 \%$. Ultrasound: occult $7 \%$, intraductal lesion $13 \%$, microcystic lesion $13 \%$, solid lesion $53 \%$, illdefined echo poor focus $13 \%$. MRI: nonmass-like nodular enhancement in a ductal, segmental or regional distribution $83 \%$, amorphous nonmasslike enhancement $17 \%$. MRI best depicted the true extent of disease.

Conclusion: Recognition of these imaging features is important for accurate surgical planning. MRI has an important role in accurately delineating disease extent and should be considered in treatment planning for NC-DCIS.

\section{References}

1. Mesurolle B, et al: Mammographically non-calcified ductal carcinoma in situ: sonographic features with pathological correlation in 35 patients. Clin Radiol 2009, 64:628-636.

2. Raza $S$, et al: Pure ductal carcinoma in situ: a range of MRI features. AJR 2008, 191:689-699.

\section{P38}

Comparison of recall, biopsy, and cancer detection rates in the Southern Derbyshire screening programme between 2006 and 2009 using hard-copy mammography and in 2009 to 2012 following the full introduction of soft-copy reporting

AE Turnbull, S Puri, M Bagnall, J York, S Farmer, N Horsley

Breast Unit, Royal Derby Hospital, Derby, UK

Breast Cancer Research 2012, 14(Suppl 1):P38

Introduction: Our programme underwent phased implementation of digital mammography, commencing soft-copy reading (SCR) in April 2009. Prior to this, all mammograms -a mixture of direct digital (DDM), computed radiography (CR) or film screen (FS) - were printed and read on multiviewers with the old films. For SCR, prior screens were available to readers but not digitised or mounted. This study was performed to evaluate recall rates, cancer detection and mammographic features assessed and biopsied, before and after SCR was introduced.

Methods: Screening episodes between 1 April 2006 and 31 March 2012 were interrogated on NBSS. Women aged 50 to 70 years were included. Screening method, soft-copy or hard-copy reading, women recalled, cancers detected and all biopsies were analysed.

Results: A total of 128,544 screening episodes were recorded in 6 years. The overall recall rate was identical at $2.5 \%$ between hard-copy and soft-copy reading. Seventy-two per cent of assessed women underwent biopsy in 2006 to 2009 and $79 \%$ in 2009 to 2012 . The cancer detection rate was identical at 8.5/1,000 in 2006 to 2009 and 8.54/1,000 in 2009 to 2012. The number of biopsies performed was 18/1,000 women screened for FS, 18.5/ 1,000 for CR and 20/1,000 for DDM. No significant difference was shown in the benign/malignant ratio of calcifications biopsied in women recalled to assessment in 2006 to 2009 compared with 2009 to 2012.

Conclusion: No significant difference was demonstrated before and after the introduction of SCR in recall rates or cancer detection. There was a nonsignificant trend towards more biopsies being performed with SCR but without additional cancers detected. Overall cancer detection rate remained high with low recall rates since adopting digital screening.

P39

Assessment of the dynamic range in dynamic contrast-enhanced magnetic resonance imaging breast examinations AEW Ledger ${ }^{r^{*}}$, M Borri ${ }^{1}, M$ Schmidt ${ }^{1}$, R Pope $^{2}$, E Scurr ${ }^{1}$, T Wallace $^{1}$,

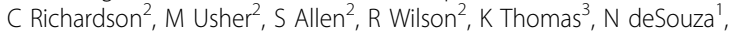
MO Leach

${ }^{1}$ CR-UK and EPSRC Cancer Imaging Centre, Institute of Cancer Research and Royal Marsden NHS Foundation Trust, Sutton, UK; ${ }^{2}$ Department of Radiology, Royal Marsden Hospital, Sutton, UK; ${ }^{3} \mathrm{Clinical}$ Research and Development,

Royal Marsden Hospital, Sutton, UK

Breast Cancer Research 2012, 14(Suppl 1):P39

Introduction: Accurate dynamic contrast-enhanced magnetic resonance imaging (DCE-MRI) protocol evaluation is necessary to ensure reliable classification of contrast-agent (CA) uptake curves. This work presents a novel retrospective method to assess the dynamic range that constructs $C A$ uptake curves using enhancement of the internal mammary artery.

Methods: Routine clinical breast examinations were performed using 3D fat-suppressed spoiled gradient-echo sequences (1.5T). Retrospective analysis was approved by the Clinical Audit Committee. Five different protocols were evaluated $\left(10^{\circ}, 14^{\circ}\right.$ and $18^{\circ}$ flip angles (FAs), radial or linear $\mathrm{k}$-space sampling), with seven to 10 patients in each group $(n=45)$. CA uptake curves were constructed from a standardised axial slice through the right mammary artery, and maximum relative enhancement $\left(E_{\max }\right)$ and timeto-peak enhancement $\left(T_{\max }\right)$ were measured for each examination [1]. $E_{\max }$ and $T_{\max }$ were compared between protocols (ANOVA/Mann-Whitney; $P<$ 0.05 indicating significance). For each protocol, calculated values of 
maximum relative enhancement $\left(E_{c a l c}\right)$ were derived from the Bloch equations and compared with $E_{\max }$ to validate the results.

Results: A lower FA and radial $k$-space sampling resulted in a statistically significant decrease in $E_{\max }(P<0.0001$ and $P=0.001$, respectively). Radial protocols exhibited greater $T_{\max }$ than linear protocols at FAs of both $14^{\circ}(P=0.025)$ and $18^{\circ}(P<0.0001)$. $E_{\text {calc }}$ was found to increase with FA as expected, with good agreement between $E_{\text {calc }}$ and $E_{\text {max }}$.

Conclusion: Significant differences were found between patient groups with only small alterations in protocol. Observations agreed with expected results, validating this method of retrospective analysis. The dynamic range is optimised at higher flip angles and with linear k-space sampling. Reference

1. Kuhl, et al: Radiology 1999, 211:101-110.

\section{P40}

Use of ultrasound in the assessment of screen-detected malignant microcalcifications

MZ Mvere*, EJ Cornford, JJ James

Nottingham Breast Institute, Nottingham, UK

Breast Cancer Research 2012, 14(Suppl 1):P40

Introduction: The aim of this study was to assess radiological and pathological features associated with successful ultrasound-guided biopsy of malignant microcalcifications.

Methods: Screen-detected breast cancers were reviewed. Cases where microcalcification was the predominant mammographic feature were identified. Ultrasound findings, mammographic appearances, method of biopsy and pathological features were recorded.

Results: There were 348 breast cancers diagnosed in the 24-month study period, 84 cases had microcalcification as the predominant mammographic feature. In 75 cases (89\%) an ultrasound scan was performed. Forty-one cases had an ultrasound abnormality and underwent an ultrasound-guided core biopsy. Successful ultrasound-guided core biopsy of malignant microcalcifications was associated with a mammographic size $>20 \mathrm{~mm}(P=$ $0.002)$, the presence of an associated mammographic feature $(P=0.03)$ and invasive disease on surgical resection $(P=0.009)$. There was no association with calcification morphology, mammographic background pattern or the histological grade of the DCIS. When the ultrasound was normal or the ultrasound-guided core biopsy inconclusive, the patient underwent an additional biopsy typically stereotactic vacuum biopsy. In the 41 cases where ultrasound-guided core was the first biopsy performed, nine cases underwent an additional biopsy prior to the therapeutic surgical procedure, compared with just one case when stereotactic vacuum biopsy was the first procedure $(P=0.005)$.

Conclusion: Ultrasound-guided core biopsy of malignant microcalcifications is more likely to be successful when mammographic size is larger and invasive disease is present. When ultrasound-guided biopsy is the first-line procedure a second biopsy is significantly more likely prior to therapeutic surgery.

\section{P41}

Comparing the use of PGMI scoring systems used in the UK and Norway to assess the technical quality of screening mammograms: a pilot study

M Boyce ${ }^{1 *}$, R Gullen ${ }^{2}$, D Parashar ${ }^{3}$, K Taylor $^{1}$

${ }^{1}$ Cambridge Breast Unit, Cambridge University Hospitals NHS Foundation

Trust, Cambridge, UK; ${ }^{2}$ Oslo Universitetssykehus, Ullevål, Oslo, Norway;

${ }^{3}$ Cambridge Cancer Trials Centre, Cambridge University Hospitals NHS

Foundation Trust, Cambridge, UK

Breast Cancer Research 2012, 14(Suppl 1):P41

Introduction: The UK and Norway use PGMI scoring to critique mammographic image quality (IQ). PGMI comprises categories with associated criteria for determining mammograms Perfect, Good, Moderate, Inadequate. Implementation of PGMI may be variable, subjective and interpreted locally, making accurate comparison of performance across countries difficult. We compared PGMI use in Cambridge and Oslo, determining differences and possible contributory factors, enabling suggestions for future research and practice.
Methods: Digital mammograms from 112 consecutively screened women were sourced in each centre. Test sets were enriched with mammograms from each PGMI category and independently scored by four mammographers, each with $\geq 4$ years' experience, using local PGMI. Each image was individually scored P, G, M, or I. Reasons for scoring less than perfect were documented and each mammogram assigned an overall PGMI score. Test sets were exchanged and the process repeated.

Results: Cambridge uses 17 criteria for scoring mammograms less than perfect. Oslo uses similar criteria, but subcategorised, totalling 39 criteria. There is fair agreement $(\kappa=0.38)$ between centres in assigning images as acceptable overall $(P, G, M)$ but poor inter-rater agreement within and between centres in further categorising acceptable mammograms as $\mathrm{P}, \mathrm{G}$ or $\mathrm{M}(\kappa<2)$. Most common faults in Oslo were skin folds, and inadequate pectoralis muscle in Cambridge. Most faults overall were on oblique views. Conclusion: Poor rater agreement and differing faults may be due to the variation in number and interpretation of categories used. Radiographer training may also be an issue. Further research should establish quantitative assessment methods and internationally uniform practice.

\section{P42}

MRI for PIP implant rupture: appearances and rupture rate

$\checkmark$ Helyar, S McWilliams, C Burke, G Charles-Edwards

Guy's and St Thomas' NHS Foundation Trust, London, UK

Breast Cancer Research 2012, 14(Suppl 1):P42

Introduction: The Poly Implant Prosthèse breast implants contain an unlicensed silicone and are associated with elevated rupture rate. We examined the performance of our MR breast protocol for implant assessment and quantified radiological rupture rate.

Methods: This was a retrospective review of 145 patients receiving an implant protocol MR breast between January and April 2012 at Guy's Hospital. Scans were reported by two consultant radiologists. Patient symptoms, MR findings and origin of surgery were recorded. Data were analysed with descriptive statistics.

Results: Twenty-one per cent (30/145) showed unilateral rupture, $46 \%$ $(14 / 30)$ bilateral rupture. Of those with a rupture, only four were symptomatic. Seventy-seven per cent (23/30) of ruptures were intracapsular, 67\% (20/30) showed evidence of silicone adenitis. MR findings show complete agreement with the explantation data gathered to date. Conclusion: These preliminary data support the use of our MR protocol for the assessment of breast implant integrity.

\section{P43}

Review of the Welsh mammographic screening programme for women who underwent mantle irradiation for Hodgkin lymphoma GM Edwards ${ }^{3 *}$, E Gallop-Evans ${ }^{2}$, C Jordan², G Stevens', K Gower Thomas ${ }^{1}$ Breast Test Wales, Cardiff, UK; ${ }^{2}$ Velindre Cancer Centre, Cardiff, UK: ${ }^{3}$ University of Wales Medical School, Cardiff, UK

Breast Cancer Research 2012, 14(Suppl 1):P43

Introduction: There is evidence that women who received supradiaphragmatic radiotherapy (sRT) for Hodgkin lymphoma at a young age are at increased risk of developing breast cancer. This study reports the implementation and results of the breast screening programme for such women in South Wales, which offers screening to women eight or more years following treatment.

Methods: Women were identified from the hospital Hodgkin Lymphoma and the Breast Test Wales screening databases. Those treated with sRT below the age of 36 years were included in the review (sRT range 9 to 34 years). Breast cancer and cause of death developing in the cohort were recorded. Results: Ninety-four eligible women were identified who underwent sRT between 1977 and 2004 in whom seven breast cancers developed. Three were found on self-examination, two through the Hodgkin screening programme, one through NHS screening and one had incomplete data. Sixteen women died, three from breast cancer. The mean induction period was 21.4 years between radiation and breast cancer presentation. Conclusion: This review confirms an increased incidence of breast cancer in these irradiated women many years following the therapy. The need for a nationwide screening programme is confirmed although the age at which to offer screening could be reviewed. 
P44

Fibroadenoma: to biopsy or not to biopsy? That is the question

AS Karuppiah*, WG Bugg, A Juette

Norfolk and Norwich University Hospitals NHS Trust, Norwich, UK

Breast Cancer Research 2012, 14(Suppl 1):P44

Introduction: In 2011, our institution adopted the policy (in line with national guidance) not to biopsy breast masses in patients $<25$ years if they have benign characteristics on ultrasound. This retrospective audit was performed prior to this decision. We compare the histology result with the USS findings of all patients $<25$ years who had a breast mass biopsied or excised, between 2003 and 2009, with respect to Stavros criteria.

Methods: Cases were identified from the Histopathology databases. Inclusion criteria include patients $<25$ years with a breast core biopsy or excision specimen. The ultrasound images were reviewed blind by a consultant breast radiologist and two registrars using Stavros criteria. The team knew one case; a blinded opinion from other consultant breast radiologists was sought.

Results: The cohort included 109 cases, of which two were malignant, three were benign phyllodes and 104 were fibroadenomata. Twenty-three out of 109 cases did not meet Stavros criteria for a benign lesion, which included one malignant spindle cell tumour, one phyllodes and 21 fibroadenomata. The one case known to the team was reviewed blind by a consultant survey and the consensus opinion was that this did not meet Stavros criteria. Eighty-five out of 109 cases met Stavros criteria for a benign lesion. Of these, 52 cases had a core biopsy and 33 cases had a surgical excision biopsy. Conclusion: All nonbenign cases were correctly identified by all reviewers using Stavros criteria. No malignancies were missed in this series. This audit suggests that the biopsy rate can be safely reduced in those $<25$ years, if the national guidance is followed.

P45

False negative assessments: an effective quality assurance method

DD Manuel*, BJG Dall, N Sharma

Leeds Teaching Hospital NHS Trust, Leeds, UK

Breast Cancer Research 2012, 14(Suppl 1):P45

Objective: To identify the frequency and characteristics of false negative assessment (FNA); an interval cancer with prior recall to assessment.

Methods: A retrospective audit, over 7 years, between 1 April 2004 and 31 March 2011. Using NBSS databases, we recorded: lesion type, size, further mammographic views, ultrasound, clinical findings, biopsy results and final histology.

Results: Twenty-nine cases by QARC, 13 true FNA and 16 excluded because contralateral or in a different quadrant. In this period, 220,522 woman were screened and 10,391 $(<5 \%)$ were referred for assessment. Total cancers detected in this period were 2,343, of which 1,867 were diagnosed at assessment and $476(20 \%)$ were interval cancers. True FNA: $2.7 \%$ of all interval cancers. True FNA: $0.6 \%$ of all screen-detected cancers. All cases were background mixed density. True FNA: 11/13 incident screen, 2/13 prevalent. Asymmetry/stromal deformity in 7/13 (54\%), calcification 4/13 (30\%), mass $2 / 13(15 \%)$. Two cases were early recall (EC) (asymmetry, and mass with calcifications), both incident screens. EC did not contribute in either case as it led to false reassurance. All asymmetry/stromal deformity reassuring on further views, with no correlating ultrasound or clinical abnormality. On review of all cases, only $2 / 13$ were felt not to have had complete triple assessment. Final pathology: two DCIS, four lobular cancers, seven ductal cancers. Seven cases were discussed at a multidisciplinary meeting.

Conclusion: True FNA remains very low. This is because assessment cases have complete triple assessment and MDT discussion. FNA can be used as a valuable educational process and mechanism to ensure consistency and adherence to NHSBSP standards.

P46

Benign appearing breast lesions age 30 and below: to biopsy,

or not to biopsy?

AC Nisbet, ${ }^{*}$ R Bradley

NHS Lothian, Edinburgh, UK

Breast Cancer Research 2012, 14(Suppl 1):P46
Introduction: Currently, most women under 30 with solid breast lumps undergo biopsy, despite classic benign features on ultrasound. However, breast cancer is uncommon in this age group and the vast majority have obvious sinister features on ultrasound. There is a growing argument that many of these younger women are being subjected to an unnecessary invasive test with obvious resource and financial implications.

Methods: A retrospective review of images and reports of all the diagnosed breast cancers in women aged 30 and below in the Edinburgh area between 2006 and 2011.

Results: A total of 37 cancers were detected in women of the sample age group. Of these, 35 had definable masses seen on ultrasound, all of which were considered R3 or above, and none of which on imaging review met benign criteria. The remaining two cancers were occult on ultrasound with no definable mass.

Conclusion: Over a 5-year period, no breast cancers would have been missed in our unit if a policy not to biopsy classic benign solid breast lesions in women aged 30 and below had been implemented. Although only looking at small numbers, this study adds to the growing evidence that biopsies of lesions with classic benign features in women of this age group are extremely unlikely to result in malignant pathology, and that current policy to routinely biopsy may be unnecessary.

\section{P47}

Classification of interval breast cancers and their presentation since screening: impact on long-term survival

YF Fong ${ }^{1 *}$, J Evans ${ }^{2}$, D Brookes ${ }^{2}, K$ Gower Thomas ${ }^{1,2}$

${ }^{1}$ Royal Glamorgan Hospital, Llantrisant, UK; ${ }^{2}$ Breast Test Wales, Cardiff, UK

Breast Cancer Research 2012, 14(Suppl 1):P47

Introduction: Interval cancers (IC) present between screenings. All interval cancers within Breast Test Wales (BTW) are classified into true interval (TI), false negative (FN), occult (OCC) and unclassified (UCC). We aim to evaluate the overall number of IC within BTW and their presentation since screening and the impact on long-term survival.

Methods: All women with IC having had screening between 1998 and 2001 were included. Classifications of IC were performed during the IC review process. Date of screening and date of diagnosis were used to calculate the interval between screening and diagnosis. Ten-year survival data were obtained on all interval cancers from national register.

Results: Some 692 interval cancers were diagnosed following screening. In total, $57.8 \%$ (391) were TI, $17.7 \%$ (120) were $\mathrm{FN}, 10 \%$ (68) were OCC and $14.5 \%$ were UCC. A total of $15.6 \%$ (108) presented within 1 year of screening, $38.2 \%$ (264) in the second year, and $46.1 \%$ (319) in the third year since screening. The overall 10 -year survival was $72.4 \%$. It was $77.5 \%$ for $\mathrm{TI}$ and $55 \%$ for $\mathrm{FN}$. The odds ratios for cancer presenting in the second year and third year were $1.009(P=0.97)$ and $1.1162(P=0.486)$, respectively, as compared with IC presenting within the first year.

Conclusion: The majority of $\mathrm{IC}$ were $\mathrm{TI}$ and presented in the third year since screening. FN had worse 10-year survival compared with TI. IC presenting in the third year did not have a statistically significantly worse long-term survival. Reduction of the screening interval would not have an impact on long-term survival outcome.

\section{P48}

Computer-aided detection of screening breast cancer: a novel approach based on genetic programming

F Canavan ${ }^{1 *}$, S Harding ${ }^{2}$, L Gustard ${ }^{3}$, AM Murphy ${ }^{3}$, JF Miller ${ }^{4}$, SL Smith $^{4}$

${ }^{1}$ Betsi Cadwaladr University Health Board, Bangor, UK; ${ }^{2}$ www.

machineintelligence.co.uk, York, UK; ${ }^{3}$ York Teaching Hospital NHS

Foundation, York, UK: ${ }^{4}$ University of York, UK

Breast Cancer Research 2012, 14(Suppl 1):P48

Introduction: Mammography is the keystone of breast cancer screening. Yet high sensitivity is achieved at the cost of low specificity - only one-third of patients recalled will have breast cancer. Computer-aided detection (CAD) is a potentially valuable tool for assisting the breast radiologist to improve positive prediction values. However, to date, CAD has not reliably altered screening outcomes and the large proportion of false positives remains a drawback. We describe a novel method to improve CAD performance called 
Cartesian Genetic Programming (CGP); a machine-based learning algorithm, akin to genetic evolution.

Methods: A population of 12 CAD programs underwent repeat fitness evaluation of how each performed in classifying breast masses. Each performed a different combination of image manipulations on 26 training mammograms. Output was subjected to a threshold, to produce a binary image predicting a benign or suspicious mass. This was compared with the image labelled by the screening radiologist. The program fitness was determined by accuracy of prediction. Fitter programs were copied and mutated to produce new variants that were re-tested. Multiple programs emerged by evolution and predictions summed to give a single more confident prediction. The confidence level was overlaid as a colour map on the original mammograms.

Results: The false positive rate was $7 / 26$ (27\%), comparing favourably with current mammography CAD systems (true positives, 13/26; true negatives, 4/26; false negatives, 2/26).

Conclusion: Our pilot study suggests CGP holds great promise for developing a viable CAD system more suited to breast screening and so warrants further evaluation.

P49

Abstract withdrawn

Breast Cancer Research 2012, 14(Suppl 1):P49

\section{P50}

Preoperative ultrasound and FNA in the diagnosis of axillary involvement in invasive breast cancer: correlation with intraoperative one-stop nucleic acid amplification and final histology

EK Jackson", N Howes, A Jones, Z Rayter, A Valencia

Bristol Royal Infirmary, Bristol, UK

Breast Cancer Research 2012, 14(Suppl 1):P50

Objective: To assess our preoperative pickup of malignant axillary lymph nodes by ultrasound and FNA compared with one-stop nucleic acid amplification (OSNA) and final histology.

Methods: At our unit all patients with invasive breast cancer undergo axillary ultrasound, and those with suspicious or equivocal findings undergo axillary FNA. If FNA is positive we proceed to axillary node clearance (ANC). If axillary assessment is normal we perform sentinel node biopsy (SNB) with intraoperative OSNA, unless planned for neoadjuvant chemotherapy or primary medical therapy. In patients with positive OSNA, further surgery is performed as per hospital protocol. Retrospective correlation of preoperative axillary ultrasound and FNA findings with intraoperative OSNA in the SNB group and final histology in the ANC group was performed, with patients diagnosed with invasive breast carcinoma between September 2010 and September 2011.

Results: See Figure 1. Seventy-seven patients were lymph node-positive $(\mathrm{LN}+\mathrm{ve})$ overall $(77 / 185=42 \%$ population $\mathrm{LN}+\mathrm{ve})$. Twenty-seven patients had preoperative LN+ve diagnosis with USS and FNA $(27 / 77=35 \%$ of $\mathrm{LN}+$ ve patients have preoperative diagnosis). Imaging of the OSNA macromet subset is reviewed and examples discussed ( $n=27: 22$ imaging normal, three equivocal, two abnormal).

Conclusion: This is the first study correlating preoperative imaging with OSNA. Our high rate of preoperative diagnosis is encouraging, but suggestions for improvement are discussed.

\section{P51}

Breast screening with magnetic resonance imaging in high-risk women L McLean $^{1 *}$, F Douglas $^{2}, \mathrm{~N}$ Forester ${ }^{1}$, CE Holmes $^{1}$

'Breast Screening Unit, Newcastle, UK; ${ }^{2}$ Department of Genetics, Newcastle, UK

Breast Cancer Research 2012, 14(Suppl 1):P51

Introduction: The Cancer Reform Strategy [1] and NICE guidelines [2] highlighted the need to identify women at increased genetic risk of breast cancer. Following this, a magnetic resonance imaging (MRI) screening service was established in Newcastle.

Methods: Women were identified from the Regional Genetic Centre database, following assessment by a consultant geneticist as high risk according to the NICE guidelines [2]. Screening comprised annual MRI \pm mammography, depending on their age, following the standards set by the NHSBSP [3]. All investigations were double-read by NHSBSP-compliant radiologists.

Results: A total of 142 women underwent 311 screening episodes between January 2008 and June 2012. There were 28 recalls (9\%) for second-look ultrasound in 24 women. Thirteen lesions had a core biopsy, from which six (1.9\%) malignancies were identified of varying histological type, size and grade. Seven lesions were benign. No ultrasound abnormality was found in the remaining 15 lesions. No interval cancers have developed.

\begin{tabular}{|c|c|c|}
\hline & \multicolumn{2}{|c|}{$\begin{array}{l}322 \text { patients with invasive breast carcinoma } \\
185 \text { patients included in retrospective analysis } \\
\text { (137 pts undergoing neoadjuvant/primary medical therapy) }\end{array}$} \\
\hline $\begin{array}{l}\text { Preoperative } \\
\text { axillary status }\end{array}$ & $\begin{array}{c}\text { Positive (c5) } 27 \text { pts } \\
\downarrow \\
\mathrm{ANC}\end{array}$ & $\begin{array}{l}\text { Negative } 158 \text { patients } \\
\text { SNB with OSNA } \\
\text { Positive } 50 \text { pts Negative } 108 \text { pts } \\
\mathbf{2 3} \text { micromets } \\
\mathbf{2 7} \text { macromets } \\
\downarrow \\
\text { ANC }\end{array}$ \\
\hline
\end{tabular}


Conclusion: In our unit, high-risk MRI screening is effective and efficient, with a cancer detection rate of 0.2 per 1,000 women screened and no false negative screens to date, with an acceptable recall rate for second-look ultrasound (within the standard set under the NHSBSP guidelines). This has been possible due to the close liaison between the departments of Genetics and Breast Screening.

References

1. Cancer Reform Strategy London: Department of Health 2007.

2. National Collaborating Centre for Primary Care: Familial Breast Cancer: The Classification and Care of Women At Risk of Familial Breast Cancer in Primary, Secondary and Tertiary Care (Partial Update of NICE Clinical Guideline 14) London: NICE 2006, 1-75.

3. NHS Cancer Screening Programmes: Technical Guidelines for Magnetic Resonance Imaging for the Surveillance of Women at Higher Risk of Developing Breast Cancer. NHSBSP Publications No. 682012.

\section{P52}

A radiological and pathological analysis of screen-detected and interval-detected breast cancers in Belfast

J Malloy ${ }^{1 *}$, C Hennell ${ }^{1}$, L Bamford ${ }^{1,2}$, K Lowry $^{1,2}$, L Tong ${ }^{1,2}$

${ }^{1}$ Belfast City Hospital, Belfast, UK; ${ }^{2}$ Northern Ireland Breast Screening

Programme (Eastern Unit), Belfast, UK

Breast Cancer Research 2012, 14(Suppl 1):P52

Introduction: The Belfast Breast Screening Programme serves a population of approximately 25,000 patients. We aimed to analyse radiological and pathological trends between screen-detected and interval breast cancers, and determine our screening lesion miss rate.

Methods: Using the Quality Assurance Reference Centre (QARC) database patients were identified with screen-detected or interval breast cancers diagnosed via the Belfast Breast Screening Programme over a fixed period. Film packs and operative specimen reports were analysed for radiological and pathological data.

Results: Screening identified $75 \%$ of breast cancers. Only $30 \%$ of screendetected lesions were palpable by breast clinicians. Sixty-one per cent of lesions were in the upper outer quadrant, with equal left-right distribution. Radiological measurements underestimated lesion size by $22 \%$. There was moderate correlation between lesion size and lymph node status. No other correlations were identified. Twenty-three per cent of interval cancers presented in year 1 following screening, 28\% in year 2 and $49 \%$ in year 3. They were larger at presentation than screen-detected cancers $(29.4 \mathrm{~mm}$ vs. $18.2 \mathrm{~mm}$ mean size) and of pathologically higher grade (39\% vs. $13 \%$ grade III). Screen-detected cancers were mostly IDC (63\%) or DCIS (18\%) subtype. Interval lesions were predominantly IDC subtype (87\%). Interval lesions showed more nodes positive per patient. Retrospective review of past screening films of interval-detected cancers showed suspicious features present in $17 \%$ of cases.

Conclusion: Breast screening identifies three-quarters of breast cancers in the screening population. Interval cancers present with increasing frequency through the screening cycle and are faster growing, pathologically more aggressive lesions.

P53

Abstract withdrawn

Breast Cancer Research 2012, 14(Suppl 1):P53

Abstract not submitted for online publication

\section{P54}

Indications for marker clip in the setting of

neoadjuvant/neoendocrine therapy

ME Fletcher*, BJG Dall, N Sharma

Leeds Teaching Hospital NHS Trust, Leeds, UK

Breast Cancer Research 2012, 14(Suppl 1):P54

Introduction: We are aware that the use of neoadjuvant therapy (NACT) and neoendocrine therapy (NAET) is steadily increasing and more patients are being referred for marker clip placement. This is our experience of the indications for marker clip placement in a large cancer unit using both NACT and NAET for treatment of breast cancers.

Methods: An 18-month retrospective audit was performed to see which patients were having marker clip placement. We recorded the indication for marker clip, final surgery and whether the marker clip was used for localisation.

Results: Fifty patients of the 92 had marker clip placement, of which 37 had NACT and 13 NAET. The marker clip was placed in 35/50 as radiology raised the possibility of near or complete pathological response. In 8/50 the marker clip was placed to aid the pathologist because of concern regarding residual low-volume disease in patients having WLE or mastectomy and 7/50 were trial patients. Thirty-eight patients had WLE and the marker clip was localised in $6 / 38$ cases. Ten patients had mastectomy and in two cases no surgery was performed due to proven metastatic disease. Nine patients had complete pathological response.

Conclusion: Due to NACT and NAET, radiologists and pathologists are facing new challenges in localising and identifying residual low-volume disease following completion of treatment. Marker clip placement can play a crucial role in ensuring accurate localisation at the time of surgery and can aid identification of residual disease for the pathologist.

\section{P55}

Should the breast be a check area on standard CT thorax examinations?

NL Marshall", L Duddy, J Barry, R Murphy, P Smiddy, M Ryan, D Hill

University College Cork, Ireland

Breast Cancer Research 2012, 14(Suppl 1):P55

Introduction: With increasing cross-sectional imaging, incidental findings (IF) are common across all subspecialities. There are implications for patients and the health service consequent to IFs: anxiety, further radiation and biopsy. Our purpose was to evaluate the malignant assessment (MA) rate for patients referred to the symptomatic breast clinic after a breast IF and to compare this with the general MA rate.

Methods: This retrospective review spanned 2 years. A trawl was performed of breast imaging reports for CT-detected lesions. Demographics, imaging and pathology results were collated. Statistical analysis using Fisher's exact test was performed to identify demographic factors associated with MAs. The MAs for the IF cohort were then compared with the general clinic population using chi-square analysis.

Results: There were 103 patients with IFs (102 women, one man) comprising 49 lesions ( 30 benign, 19 malignant). In the general service were 10,330 patients with 2,551 lesions (683 malignancies). The IF cohort were more likely to have a lesion $(47.6 \%$ vs. $28.7 \%, P<0.001)$ and to have a MA $(18.4 \%$ vs. $6.7 \%, P<0.0001)$. The only demographic factor of the IF group with a statistically significant association with MA was age $>65$ years $(P=$ 0.0063).

Conclusion: While patients with breast IFs are more likely to have a MA, only $47 \%$ of those referred actually had a lesion. Age was the only statistically significant factor that correlated with a MA. Whilst a worthy check area clinicians should exercise caution to avoid unnecessary procedures, particularly in younger patients.

P56

Node-positive breast cancers diagnosed in 2011 at York Teaching

Hospitals NHS Trust: an analysis of the adequacy of current preoperative assessment

A Chandra

York Hospital NHS Foundation Trust, York, UK

Breast Cancer Research 2012, 14(Suppl 1):P56

Introduction: Preoperative assessment of the axillary nodal status in primary breast cancer using ultrasonagraphy is now established practice and mandated by the NHSBSP. However, the criteria for nodal biopsy are based on the morphological appearance of the imaged nodes. This study's poster proposes that the tumour and grade stage of the primary tumour should also influence the threshold for nodal biopsy with the intention of improving preoperative assessment and thus decreasing morbidity 
associated with further surgical intervention to the axilla following positive sentinel lymph node biopsy.

Methods: A retrospective analysis of final nodal status of the 296 surgically treated patients diagnosed with primary breast cancer in 2011 was performed. Data including preoperative assessment of tumour size, grade, axillary node status (preoperative and postoperative) and final outcome was collected. Patients with negative preoperative axillary node status were compared with postoperative node status and the proportion requiring further treatment was ascertained.

Results: A total of 207 had negative axillary preoperative assessment and underwent sentinel lymph node biopsy (SLNB). Fifty-one had positive sentinel lymph nodes, and of these $9 \%$ required further surgery. Sensitivity at the identification of positive axillary nodal disease preoperatively was 0.49 and specificity calculated at 0.99 . Preoperative assessment had a positive predictive value of $96 \%$ and a negative predictive value of $73 \%$. A positive correlation between axillary nodal involvement and tumour size and grade was observed; that is, $15 \%$ in T1G1 tumours to $36 \%$ in T3G3 tumours. Patients in the T2G2 group or above were $54 \%$ more likely to have a positive SLNB.

Conclusion: We propose patients staged preoperatively as T2G2 and above should have axillary node biopsy considered despite negative preoperative ultrasound. This may increase the accuracy of axillary preoperative assessment with a subsequent decrease in second operations to the axilla. References

1. Aiten $E$, Osman M: Factors affecting nodal status in invasive breast cancer: a retrospective analysis of 623 patients. Breast J 2010, 16:271-278.

2. Hogan BV, Shenoy HG, et al: The use of ultrasound in pre-operative assessment of the axilla in breast cancer [abstract]. Cancer Res 2009, 69(2 Suppl).

\section{P57}

Predicting risk of malignancy in subgroups of $B 3$ breast lesions

ND Forester*, M Brotherton, A-M Wason

Department of Breast Radiology, Bradford, UK

Breast Cancer Research 2012, 14(Suppl 1):P57

Introduction: Heterogeneity and varying malignancy risk makes B3 lesion management difficult. Can histological features predict malignancy risk? Methods: A retrospective review of B3 lesions (April 2005 to March 2010) following $14 \mathrm{G}$ biopsy, followed to final pathology. Key phrases from pathology identified; atypia, radial scar/complex sclerosing lesion (RS/CSL), atypical intraductal proliferation (AIDP), atypical ductal/lobular hypertrophy $(\mathrm{ADH} / \mathrm{ALH})$, flat epithelial atypia (FEA), lobular in situ neoplasia (LISN), and so forth. Age-adjusted logistic regression to assess risk of malignancy (Stata11).

\section{Table 1(abstract P57)}

\begin{tabular}{llllll}
\hline Pathology & Number & Odds ratio & $\boldsymbol{P}$ value & $\mathbf{9 5 \%} \mathbf{C l}$ \\
\hline Atypia & 53 & 7.48 & $<0.001^{*}$ & 2.62 to 21.39 \\
AIDP & 10 & 4.87 & $0.034^{*}$ & 1.13 to 21.01 \\
ADH & 11 & 2.50 & 0.167 & 0.68 to 9.24 \\
ALH & 6 & 1.28 & 0.793 & 0.21 to 7.93 \\
FEA & 8 & 5.90 & $0.025^{*}$ & 1.25 to 27.67 \\
CCC & 31 & 5.10 & $0.001^{*}$ & 1.89 to 13.76 \\
Papillary & 14 & 0.57 & 0.44 & 0.14 to 2.38 \\
LISN & 30 & 0.55 & 0.28 & 0.18 to & 1.64 \\
RS/CSL & 13 & 0.92 & 0.91 & 0.23 to 3.74 \\
Epithelial proliferation & 20 & 2.07 & 0.172 & 0.73 to 5.88 \\
Mucocele-like & 6 & 1.91 & 0.48 & 0.32 to 11.41 \\
Fibroepithelial & 10 & No malignant diagnoses & \\
Haemangioma & 1 & No malignant diagnoses & \\
Spindle cell & 2 & No malignant diagnoses & \\
\hline
\end{tabular}

*Significant $P$ value.
Results: A total of 205 B3 lesions were identified; 112 lesions with subsequent excision biopsy were analysed. Patients had mean age of 56 years $(95 \% \mathrm{Cl}=55$ to 57 years). Thirty out of 112 lesions were upgraded to B5 diagnosis. Nine out of 112 had final diagnosis of LCIS. Multivariate analysis of odds ratios for malignancy, after age adjusting, is shown in Table 1.

Conclusion: Atypia on core biopsy significantly predicts malignancy, with 7.48 times the odds of malignancy compared with lesions without atypia $(P<0.001 ; 95 \% \mathrm{Cl}=2.62$ to 21.39$)$. Similarly, lesions containing AIDP, FEA and columnar cell change (CCC) have significantly increased odds for malignancy. LISN did not confer an increased risk of malignancy. Stratifying lesions in this way can direct future management.

\section{P58}

Breast MRI quality assurance in practice

S Bacon*, B Dall, N Sharma, D Manuel, D Wilson

St James's Institute of Oncology, Leeds, UK

Breast Cancer Research 2012, 14(Suppl 1):P58

Introduction: Breast MRI has been incorporated into the NHSBSP programme and therefore is subject to quality assurance (QA) to NHSBSP standards. The breast screening technical guidelines recommend weekly testing of signal-to-noise ratio (SNR) and suppression effectiveness. We tested a method of implementing these recommendations on a Siemens 1.5T Avanto scanner using phantoms and software supplied as standard. Methods: Phantoms were placed in the right and left apertures of the Siemens four-element breast matrix coil and manufacturer QA performed. Suppression effectiveness was measured by acquiring a 3D spoiled gradient echo (FLASH) sequence with and without water suppression. Regions of interest (ROI) were drawn in both phantoms and percentage signal reduction due to water suppression calculated for three slices. SNR was measured using the standard fat-suppressed 3D FLASH sequence acquired three times after waiting 10 minutes to minimise fluid motion artefacts. Subtractions were performed and ROI drawn in both phantoms. SNR was calculated as $\sqrt{2} \times$ (mean signal intensity)/(standard deviation of the subtraction image) for three slices.

Results: The breast coil passed the manufacturer QA tests. Mean suppression effectiveness was $97.0 \%$ (95.6 to $98.0 \%$ ) for the right phantom and $96.9 \%$ (95.4 to $97.7 \%)$ for the left. Mean SNR was 98.8 (41.2 to 110.9) for the right phantom and 96.2 (53.8 to 115.4) for the left. The low values of SNR were likely to be due to artefacts from fluid motion on the subtraction images used for noise calculations.

Conclusion: The methods proposed allowed an independent measure of suppression effectiveness. More work is needed to ensure a reliable measurement of SNR.

P59

Breast clinicians: their contribution to the breast screening programme in England and implications of the current age structure

C Swinson

Beds \& Herts Breast Screening Service, Luton, UK

Breast Cancer Research 2012, 14(Suppl 1):P59

Introduction: Since the role of breast clinician was established in 1987 to support introduction of the NHSBSP, the role has developed and breast clinicians are now important members of the MDT, skilled in the full range of screening work. With a shortage of radiologists this input must continue, but recruitment may be hindered by a restrictive contractual structure. This study set out to establish the contribution of breast clinicians to the screening programme, and their age distribution.

Methods: In July 2012 an email survey of members of the Association of Breast Clinicians was conducted to determine staffing, film-reading workload and age distribution data in English screening units. Responses were received from 16 units, around $90 \%$ of units with breast clinician filmreaders.

Results: The 16 units were mainly in the south and included some of the largest screening units. Overall, there were 30 breast clinician filmreaders, 56 consultant radiologists and 46 radiographer film-readers. Numbers of clinicians in units varied from one to five, representing 9 to $50 \%$ of all film-readers. Breast clinicians read from 9 to $66 \%$ of films, and 
in 12 units read proportionately more films than expected from their number. Twenty-two (73\%) of the clinicians were aged over 50 and $14(47 \%)$ over 55.

Conclusion: Breast clinicians make a substantial contribution to screening units in England, particularly in the south. Retirements over the next 5 to 10 years will add considerably to manpower pressures on the NHSBSP. Recruitment will be dependent upon an attractive career structure and robust training.

\section{P60}

Vacuum-assisted core biopsy of the breast: a 3-year

single-centre experience

M Al-Attar, MM Hoosein*, K Wren, D Lister, E Denton, G McDonald, S Kaneri,

L Grosvenor

University Hospitals of Leicester, UK

Breast Cancer Research 2012, 14(Suppl 1):P60

Introduction: Vacuum-assisted core biopsy (VACB) of the breast is a minimally invasive technique, used increasingly for the assessment of mammographically and ultrasound detected, nonpalpable breast lesions. The effectiveness of VACB has been demonstrated on lesions both with and without microcalcifications. VACB allows the operator to obtain a sufficient specimen with a single insertion to provide for a more accurate diagnosis.

Methods: The aim of this study was to audit the use and effectiveness of ultrasound and stereotaxis guided VACB at a single centre over a 3-year period. We retrospectively identified patients from the Leicestershire Breast Screening Service and Radiology Information Systems records. A total of 152 patients undergoing 157 VACB $(n=157)$ were identified. Of these, 133 were stereotaxis guided, the remaining 24 ultrasound guided. The overall lesion workload was as follows: asymmetrical densities $=6(4 \%)$; mass $=$ $31(20 \%)$; parenchymal distortion $=32(20 \%)$; and calcification $=88(56 \%)$.

Results: A positive histological diagnosis was achieved in 153 (97\%) of the total VACBs performed. Of these patients, $136(88.9 \%)$ had a prior $14 \mathrm{G}$ core biopsy, $16(10.5 \%)$ had VACB as a first-line procedure. Outcomes for VACB were then compared against $14 \mathrm{G}$ biopsies for individual histological grade, presented as follows - grade on $14 \mathrm{G}$ needle (definitive diagnosis percentage on VACB): B1 (83\%); B2 (67\%); B3 (62\%); B4 (76\%); and B5C (100\%). Overall, $51(33.6 \%)$ patients avoided surgery for benign breast disease $(95 \% \mathrm{CI}=$ 26.1 to $41.1 \%)$, and 33 patients (21.7\%) had single definitive surgery thereby avoiding multiple procedures ( $95 \% \mathrm{Cl}=15.2$ to $28.3 \%$ ).

Conclusion: VACB is a safe, well-tolerated and extremely useful sampling modality which can, in selective cases, accurately determine onward surgical management and avoid unnecessary surgery for benign breast disease.

\section{P61}

Screening breast MRI in high-risk women undergoing prophylactic mastectomy

SE McWilliams

Guy's and St Thomas' NHS Foundation Trust, London, UK

Breast Cancer Research 2012, 14(Suppl 1):P61

Introduction: High-risk women with genetic predisposition for breast cancer are increasingly being offered bilateral prophylactic mastectomy for risk reduction. If an incidental tumour is found at surgery, the patient cannot then undergo sentinel node biopsy of the axilla and has to have a full axillary clearance associated with morbidity of lymphoedema often in young women. We look at the use of screening MRI in 16 women at our institution prior to surgery to see how this affected management.

Methods: Sixteen women aged 25 to 33 years underwent breast MRI prior to prophylactic mastectomy. The women were high risk with proven BRCA 1 or 2 mutations. The images were independently double-reported by two certified board breast radiologists. The patients also underwent bilateral mammogram.

Results: Of the 16 women, one patient had a small tumour selected on MRI that was occult on conventional imaging and was able to undergo sentinel node biopsy. In the remaining 15 patients, one small $3 \mathrm{~mm}$ lesion was found at surgery and the patient had to have axillary dissection.

Conclusion: Prophylactic mastectomy precludes the use of sentinel node biopsy as the breast is required for this procedure in order for the dye and tracer to be injected into the breast. Screening MRI prior to prophylactic mastectomy is essential in order for a sentinel node to be feasible in case a small tumour is present occult on conventional imaging.

\section{P62}

Mammograms in 35-year-old to 39-year-old symptomatic patients

KA Lim*, P Young

Cardiff and Vale University Health Board, Cardiff, UK

Breast Cancer Research 2012, 14(Suppl 1):P62

Introduction: Currently, in our institution, all women presenting at the Triple Assessment Symptomatic Breast Clinic over 35 years of age undergo two-view mammograms. Recent recommendations suggest that mammograms should only be performed above the age of 40 [1]. We reviewed our patients to see whether changing our policy would have resulted in any missed cancers.

Methods: All patients on our Breast Cancer Database diagnosed between April 2002 and March 2012, aged between 35 and 39 years at diagnosis, were identified. Patient records were reviewed to determine how the cancer was diagnosed and treated.

Results: Fifty-one patients were identified; 11 were diagnosed elsewhere, and therefore excluded, and one was screen-detected and also excluded. Of the remaining 39 patients, one patient was male. In 33/39 (84.6\%) patients, ultrasound was performed of a palpable lesion and mammography did not add any additional diagnostic value. Of the remaining six patients, three had obvious clinical signs of malignancy. The remaining three patients had malignancy diagnosed as the result of their mammograms. Two (5.1\%) had mammographic microcalcification due to intermediate-grade DCIS and one patient had an impalpable cancer, seen on the mammogram, which prompted the diagnostic ultrasound.

Conclusion: In our institution, over a 10-year period, two cases of intermediate-grade DCIS and one case of Grade 2 invasive ductal carcinoma would have been missed if routine mammography had not been performed in the 35 to 39 year age group.

Reference

1. Best Practice Diagnostic Guidelines for Patients Presenting with Breast Symptoms London: Department of Health: Willett AM, Michell MJ, Lee MJR 2010.

Cite abstracts in this supplement using the relevant abstract number, e.g.: Lim and Young: Mammograms in 35-year-old to 39-year-old symptomatic patients. Breast Cancer Research 2012, 14(Suppl 1):P62 